\title{
Research on Vision System Calibration Method of Forestry Mobile Robots
}

\author{
Ruting Yao, Yili Zheng, Fengjun Chen*, Jian Wu, Hui Wang, \\ The School of Technology, Beijing Forestry University, \\ Beijing 100083, China \\ *corresponding author's email: chenfj227@bjfu.edu.cn
}

Received: November 24. Revised: December 29, 2020. Accepted: December 29, 2020.

\begin{abstract}
Forestry mobile robots can effectively solve the problems of low efficiency and poor safety in the forestry operation process. To realize the autonomous navigation of forestry mobile robots, a vision system consisting of a monocular camera and two-dimensional LiDAR and its calibration method are investigated. First, the adaptive algorithm is used to synchronize the data captured by the two in time. Second, a calibration board with a convex checkerboard is designed for the spatial calibration of the devices. The nonlinear least squares algorithm is employed to solve and optimize the external parameters. The experimental results show that the time synchronization precision of this calibration method is $0.0082 \mathrm{~s}$, the communication rate is $23 \mathrm{~Hz}$, and the gradient tolerance of spatial calibration is $8.55 \mathrm{e}-07$. The calibration results satisfy the requirements of real-time operation and accuracy of the forestry mobile robot vision system. Furthermore, the engineering applications of the vision system are discussed herein. This study lays the foundation for further forestry mobile robots research, which is relevant to intelligent forest machines.
\end{abstract}

Keywords-Systems Theory, Signal processing, Imaging, Forestry mobile robots, Vision system, Time synchronization, Extrinsic calibration

\section{INTRODUCTION}

$\mathrm{A}$ CCELERATING the development of technology and equipment is an essential approach to transform the mode of forestry production [1]. Forests have harsh environments and require high labor intensity. The use of mobile robots to replace manpower can effectively reduce labor costs and potential safety hazards in forestry work. The vision system is the core of a forestry mobile robot, and its calibration affects the ability of the robot to perceive the environment. The vision system of forestry mobile robots must satisfy the real-time operation and accuracy requirements [2],[3]. To satisfy the requirement of real-time operation, the vehicle running speed is assumed to be $40 \mathrm{~km} / \mathrm{h}$, and the operating frequency of the vision system should exceed $10 \mathrm{~Hz}$. The vision system accuracy is related to the accuracy of the sensor, internal and external parameter calibrations, and the vision processing algorithm [4].
The study of vision systems for the forest operation process has attracted the interest of many researchers, leading to a certain extend of progress. In developing the forestry vision system, researchers initially used LiDAR or a camera. LiDAR can be used to detect the diameter and position of trees in forest environments [5]-[8], and a camera can capture the color and texture features of the forest environment for detection and classification [9]-[12]. A three-dimensional (3D) LiDAR can obtain more complete shape and position information, but it is highly expensive. In contrast, a camera is inexpensive for capturing the color and texture of the environment, but it cannot detect information such as position, shape, and size. Compared with the 3D LiDAR, a two-dimensional (2D) LiDAR is less expensive, but its point cloud is in a single line[13]. Nevertheless, the foregoing shortcomings can be effectively compensated by the combined use of a camera and 2D LiDAR.

The joint calibration between the camera and 2D LiDAR affects the perception of the vision system. If the data obtained by both devices are calibrated and processed, effective 3D information can be obtained. The joint calibration of camera and 2D LiDAR information was first proposed by Qilong Zhang and Robert Plesst [14]. Based on the observation of an external checkerboard, they extracted LiDAR points and checkerboard grid points to optimize the external geometric parameters. Jae-Yeul Kim and Jong-Eun Ha completed the extrinsic calibration of the camera and 2D LiDAR by adding a dummy camera to remove IR cut filter [15]. In addition, the corresponding relationship between these two devices was determined based on a calibrated camera image to obtain external parameters. Van-Dung Hoang created a calibration plate consisting of two adjacent and discontinuous right triangles that could automatically extract the corresponding points between the two sensors to estimate the external parameters between them using a matrix [16]. Jesus Briales extracted the orthogonal points of the LiDAR point cloud and camera image using an orthogonal trihedron and then obtained the rigid body transformation between them [17]. Khalil used a checkerboard and orthogonal planes to solve the external parameters between a camera and 2D LiDAR without overlapping [18]. Xuepeng Chu et al. used a triangular plate as a calibration tool and extracted the intersection between the 
edge of the plate and the LiDAR point cloud in the image. Then they employed the point-line correspondence to obtain calibration parameters [19]. In [20], an infrared cutting filter was first used to make the scanning points of the 2D LiDAR visible in the camera image, then edge matching and top-down methods were employed to estimate extrinsic parameters. Itami Fumio et al. proposed an improved calibration method using a checkerboard calibration board and checkerboard normal vectors to construct additional constraints between the camera and 2D LiDAR [21].

This study considered the requirements for the vision system of forestry mobile robots as a starting point in building the vision system using the combination of a monocular camera and 2D LiDAR. The camera was first calibrated based on the working principle of the devices. Then, the vision system was calibrated in time and space. Finally, the 2D LiDAR point cloud and the image were matched to obtain 3D information of the environment. These processed data establish the foundation for obtaining more accurate information and provide a basis for follow-up research on forestry mobile robots.

\section{MATERIALS AND METHODS}

\section{A. Overall structure}

An Ackerman robot equipped with an electronic compass and encoder was used as the basic platform in this study; the robot system is shown in Figure 1. Its length, width and height are 990,336 and $345 \mathrm{~mm}$, respectively; its maximum speed is $1.5 \mathrm{~m} / \mathrm{s}$. The monocular camera and 2D LiDAR are installed horizontally in the front part of the robot, constituting the vision system. The distance between the vision system and the ground is $100 \mathrm{~cm}$. The 2D LiDAR is HOKUYO UST-10LX, which can perform a $2 \mathrm{D}$ plane scan at $270^{\circ}$ within a $10 \mathrm{~m}$ radius. It scans the contour information in the surrounding environment and outputs the information via the IPV6 network interface at a rate of $40 \mathrm{~Hz}$ to form a raw point cloud. The monocular camera is LRCP10230_1080P with the resolution set to $640 \times 480$ pixels; it can transmit the color and texture of the surrounding environment to the master via the universal serial bus (USB) data interface. As shown in Figure 2, the program runs on the robot operating system (ROS) Kinetic. The overall structure includes the hardware, operating, and application layers. The hardware layer transmits the collected information to the operating system via their own communication interface. The operating system transmits the information to function packages in the application layer, and finally the functions such as obstacle detection and positioning are realized.

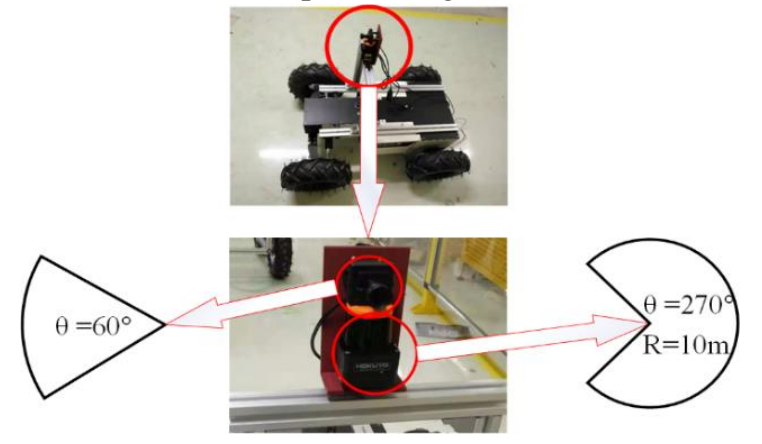

Fig.1 The system of the forestry mobile robot

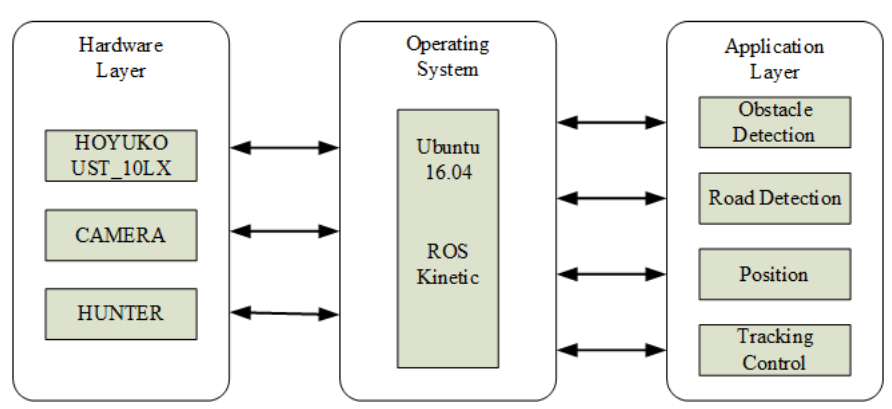

Fig.2 Overall structure of the forestry mobile robot

\section{B. Calibration tool}

In addition, a calibration plate needs to be prepared for calibrating the internal and external sensor parameters. Figure 3 shows a checkerboard calibration board with two $60 \mathrm{~mm}$ high raised cells and $12 \times 9$ checkerboard squares of a size of $20 \mathrm{~mm}$ $\times 20 \mathrm{~mm}$ each. To determine the feature points effectively, it must be ensured that the measured plane of the LiDAR and the two raised cells are on the same plane during calibration. The camera can determine each feature point on the checkerboard by observing the calibration board. And the feature points of the two raised cells on the calibration board are determined. Therefore, the relevant feature points in the $2 \mathrm{D}$ point cloud and image can be determined.

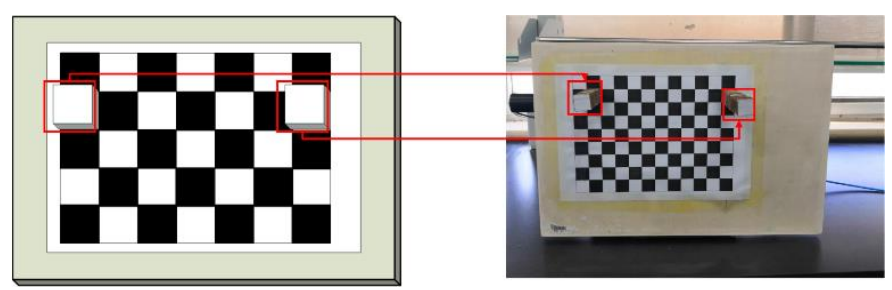

Fig.3 Checkerboard calibration plate with two raised cells

\section{Camera calibration}

In this study, the camera used is a pinhole model charge-coupled device (CCD). Zhang's calibration method [22], whose calibration tool is checkerboard, was employed. Compared with other methods, this calibration method has a lower cost, higher precision and robustness. The calibration model of the internal parameters of the camera is expressed as follows:

$$
\begin{gathered}
\mathrm{s}\left[\begin{array}{c}
\mathrm{u} \\
\mathrm{v} \\
1
\end{array}\right]=\left[\begin{array}{ccc}
\mathrm{f}_{\mathrm{x}} & 0 & \mathrm{c}_{\mathrm{x}} \\
0 & \mathrm{f}_{\mathrm{y}} & \mathrm{c}_{\mathrm{y}} \\
0 & 0 & 1
\end{array}\right]\left[\mathrm{r}_{1} \mathrm{r}_{2} \mathrm{t}\right] \cdot\left[\begin{array}{c}
\mathrm{X} \\
\mathrm{Y} \\
1
\end{array}\right] \\
\mathrm{K}=\left[\begin{array}{ccc}
\mathrm{f}_{\mathrm{x}} & 0 & \mathrm{c}_{\mathrm{x}} \\
0 & \mathrm{f}_{\mathrm{y}} & \mathrm{c}_{\mathrm{y}} \\
0 & 0 & 1
\end{array}\right]
\end{gathered}
$$

where: $[u, v, 1]^{T}$ is the homogeneous coordinates of the points on the calibration plate projected onto the image plane; $[X, Y, 1]^{T}$ is the homogeneous coordinate of the point on the 
calibration plate; $\left(r_{1}, r_{2}\right)$ is the rotation matrix of camera coordinate system relative to the world coordinate system; $t$ is the translation vector of camera coordinate system relative to the world coordinate system; $K$ is the internal matrix of the camera..

By setting the homography matrix to

$$
H=\left[\begin{array}{lll}
h_{1} & h_{2} & h_{3}
\end{array}\right]=\lambda K\left[\begin{array}{lll}
r_{1} & r_{2} & t
\end{array}\right]
$$

the following is obtained,

$$
r_{1}=\frac{1}{\lambda} K^{-1} h_{1}, r_{2}=\frac{1}{\lambda} K^{-1} h_{2}
$$

Because $r_{1}$ and $r_{2}$ are orthogonal unit quantities, the constraint relationship between the two variables can be derived, and $K$ can be obtained using multiple sets of data. The final internal parameter of the camera is as follows:

$$
K=\left[\begin{array}{ccc}
711.6578 & 0 & 348.7275 \\
0 & 734.8004 & 294.6621 \\
0 & 0 & 1
\end{array}\right]
$$

\section{Time Synchronization}

The camera and 2D LiDAR are independent in terms of information collection. The HOKUYO UST-10LX LiDAR communicates through an IPV6 network interface with a transmission rate of $40 \mathrm{~Hz}$, whereas the $\mathrm{CCD}$ camera communicates through a serial port with a transmission rate of approximately $30 \mathrm{~Hz}$. The two devices have different communication methods and transmission rates. Consequently, the arrival times of the 2D point cloud and image that are received by the main controller do not match; hence, synchronization is necessary [23]. The timestamp corresponding to each data when the main controller received the data was recorded and used to divide the time period [25]. In this study, an adaptive algorithm [24] was used to align the timestamp.

All image messages were formed into an image queue according to time, and all $2 \mathrm{D}$ point cloud messages were formed into 2D point cloud queues. An image message and a $2 \mathrm{D}$ point cloud message received over the same time period formed a set of information. Moreover, at least one of the two adjacent sets was to have no missing messages. The latest information at the head of the two queues is named pivot. The size of the set is the difference between the latest and earliest timestamps of the set. If $\mathrm{R}$ is the latest set and $\mathrm{N}$ is the next set, then the alignment steps are shown in Figure 4. For example, when the last set is determined, the new image message I1 enters the image queue and awaits the next message. If the next message is an image message I2, then I1 is discarded and the next message is awaited. If the next message is a point cloud message L1, then L1 enters the point cloud queue and is named the pivot. The next message is awaited; if the next message is the point cloud message L2, then L1 and I1 form a new set. If the next message is the image message I3, the sizes of L1I1 and L1I3 are calculated. If the size of L1I1 is small, then L1I1 is a new set; otherwise, L1I3 is a new set.

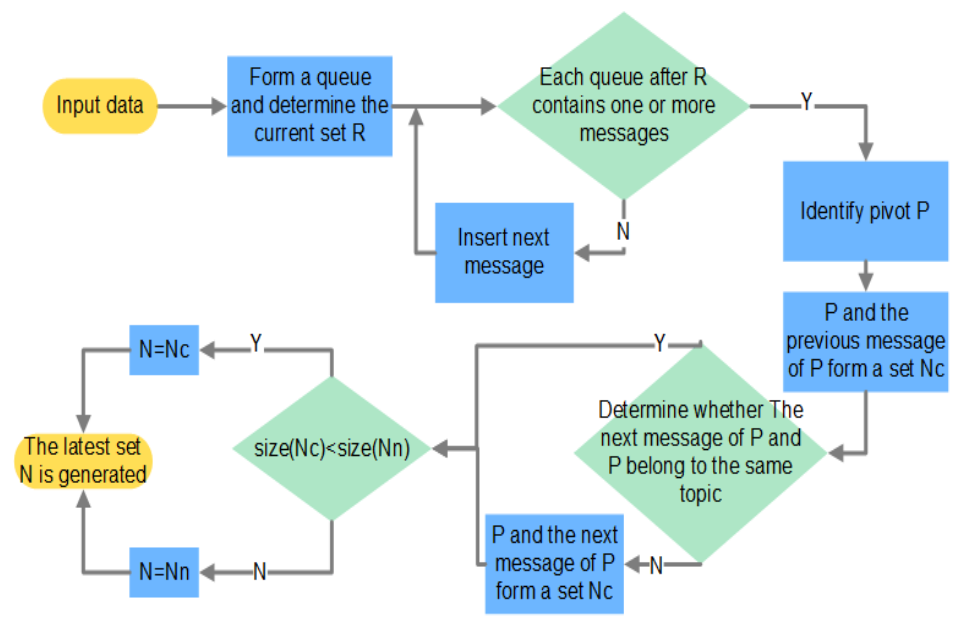

Fig.4 Time alignment steps

The orange line shown in Figure 5 represents the image queue, and each circle on the orange line represents an image message. The purple line shown in Figure 5 represents the 2D point cloud queue, and each square on the purple line represents a $2 \mathrm{D}$ point cloud message. The dark red message represents the pivot. The messages connected by dashed lines constitute a set, indicating that these two messages are in the same period. The rest of the messages are ignored.

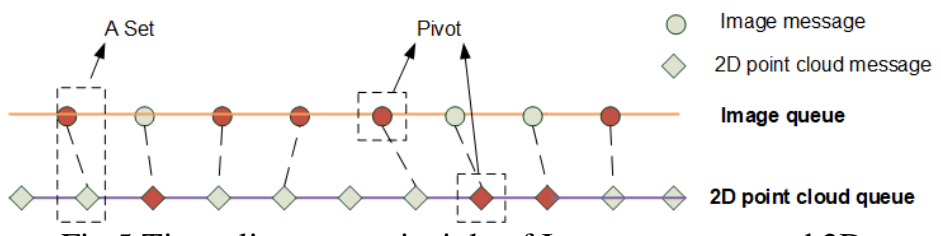

Fig.5 Time alignment principle of Image message and 2D point cloud

\section{E. Spatial calibration}

When the data collected by the camera and 2D LiDAR are combined, they must be calibrated in terms of time and spatial coordinates. The purpose of spatial registration is to obtain the external parameters [17] between the 2D point cloud and image coordinate systems and establish an effective match between the two of them, thus laying the foundation for further obtaining $3 \mathrm{D}$ information about the forest. The coordinate systems are shown in Figure 6.

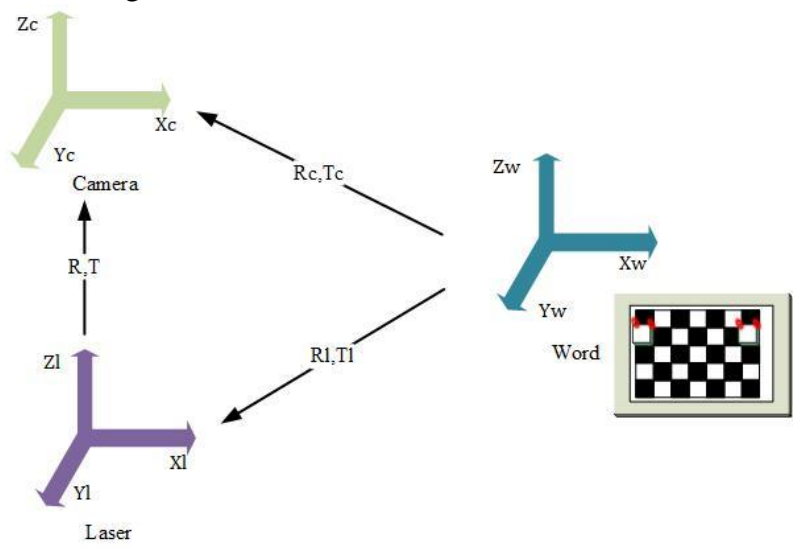


Fig.6 Coordinate system transformation

In this study, a checkerboard calibration plane with bulges was employed for externally calibrating the camera and 2D LiDAR. Suppose the feature point marked in red in Figure 6 is $P_{w}=\left[X_{w}, Y_{w}, Z_{W}\right]$, and its projection in the camera coordinate system and image coordinate system are $P_{c}=\left[X_{c}, Y_{c}, Z_{c}\right]$ and $P_{I}=[u, v]$, respectively. According to the camera calibration results, the following could be obtained:

$$
\left[\begin{array}{l}
u \\
v \\
1
\end{array}\right]=\left(k / Z_{c}\right)\left[\begin{array}{cccc}
f_{X} & 0 & c_{X} & 0 \\
0 & f_{y} & c_{y} & 0 \\
0 & 0 & 1 & 0
\end{array}\right]\left[\begin{array}{c}
X_{c} \\
Y_{c} \\
Z_{c} \\
1
\end{array}\right]
$$

The scanning plane of the LiDAR passed feature point $P_{w}$, and the projection of $P_{w}$ in the 2D LiDAR coordinate system is $P_{L}=\left[X_{l}, Y_{l}, Z_{l}\right]$. In this experiment, the laser plane was located in a horizontal plane with a height $h$, and the coordinates obtained by the $2 \mathrm{D}$ LiDAR were those of polar coordinate system. Therefore, the projection of $P_{w}$ in the 2D point cloud is

$$
P_{L}=[d \sin \alpha, d \cos \alpha, h]
$$

where: $d$ is the distance value of $P_{w}$ measured by 2D LiDAR; $\alpha$ is the angle of $\mathrm{P}_{\mathrm{w}}$ measured by $2 \mathrm{D}$ LiDAR.

The rotation matrix and translation vector between the $2 \mathrm{D}$ LiDAR and camera are denoted as $\mathrm{R}$ and $\mathrm{T}$, respectively.

$$
\begin{gathered}
R=\left[\begin{array}{lll}
\varphi_{11} & \varphi_{12} & \varphi_{13} \\
\varphi_{21} & \varphi_{22} & \varphi_{23} \\
\varphi_{31} & \varphi_{32} & \varphi_{33}
\end{array}\right] \\
T=\left[\begin{array}{l}
\Delta_{1} \\
\Delta_{2} \\
\Delta_{3}
\end{array}\right]
\end{gathered}
$$

Therefore, the relationship between the $2 \mathrm{D}$ point cloud and image coordinate systems is as follows:

$$
P_{I}=R \cdot P_{L}+T
$$

The different $P_{w}$ values were measured by setting the device at different positions and angles to obtain the corresponding arrays. Assume that the set of corresponding $P_{w}$ points in the image, and the set of corresponding $P_{w}$ points in the $2 \mathrm{D}$ point cloud are given by Eqs.(11) and (12), respectively.

$$
\begin{aligned}
& P_{I}=\left\{c_{0}, c_{1}, \ldots, c_{n}\right\} \\
& P_{L}=\left\{l_{0}, l_{1}, \ldots, l_{n}\right\}
\end{aligned}
$$

The centres of mass of $C_{i}$ and $l_{i}$ are as follows:

$$
\bar{c}=\frac{1}{N} \sum_{i=1}^{N} c_{i}
$$

$$
\bar{l}=\frac{1}{N} \sum_{i=1}^{N} l_{i}
$$

The coordinates of the de-centroid are as follows :

$$
\begin{aligned}
c_{i}^{\prime} & =c_{i}-\bar{c} \\
l_{i}^{\prime} & =l_{i}-\bar{l}
\end{aligned}
$$

The least squares problem is formulated, as given by Eq.(17) .

$$
\begin{gathered}
E=\min _{R, T} \frac{1}{2} \sum_{i=1}^{n}\left\|\left(c_{i}^{\prime}-\left(R * l_{i}^{\prime}+T\right)\right)\right\|^{2} \\
R^{*}=\arg \min _{R} \frac{1}{2} \sum_{i=0}^{n}\left\|c_{i}^{\prime}-R * l_{i}^{\prime}\right\|^{2}
\end{gathered}
$$

The singular value decomposition (SVD) [26] is used to solve Eq.(19):

$$
W=\sum_{i=1}^{n} c_{i}^{\prime} l_{i}^{\prime T}=U\left[\begin{array}{ccc}
\sigma_{1} & 0 & 0 \\
0 & \sigma_{2} & 0 \\
0 & 0 & \sigma_{3}
\end{array}\right] V^{T}
$$

where: $U$ and $V$ are orthogonal matrices; $\sigma_{1}, \sigma_{2}, \sigma_{3}$ are the singular values arranged from the largest to the smallest.

When $\mathrm{W}$ is a full rank matrix, $R^{*}$ and $T^{*}$ are as follows:

$$
\begin{gathered}
R^{*}=V U^{T} \\
T^{*}=\bar{c}-R^{*} * \bar{l}
\end{gathered}
$$

However, the foregoing are linear solutions that depend on the readings of feature points and initial values. If there was an error in reading the feature points, the errors of $\mathrm{R}^{*}$ and $\mathrm{T}^{*}$ would be large. Therefore, the method of nonlinear least squares was used to optimise the above model, thus increasing the robustness and stability of the calibration model. As shown in Eq.(22), the cost function is as follows:

$$
e_{i}=c_{i}^{\prime}-\left(R * l_{i}^{\prime}+T\right)
$$

To reduce the degree of penalty for outliers, the Huber loss

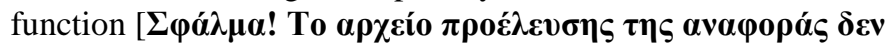

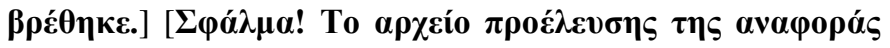

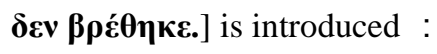

$$
H\left(e_{i}\right)=\left\{\begin{array}{l}
\frac{1}{2} e_{i}{ }^{2}, \text { when }\left|e_{i}\right|<\delta \\
\delta\left(\left|e_{i}\right|-\frac{1}{2} \delta\right), \text { other }
\end{array}\right.
$$

where: $\delta$ is the pre-set threshold r. 
When $e_{i}<\delta$, the loss function adopts the square error; otherwise, a linear error is adopted. $\delta$ is determined based on the difference between the model and actual parameters. If the difference is small, $\delta$ becomes as large as possible. Otherwise, $\delta$ shrinks as much as possible. Eq.(10) is rewritten Eq.(24).

$$
P_{I}=f\left(P_{L}\right)
$$

The difference between the model and actual parameters is defined as Eq.(25).

$$
\rho=\frac{f\left(P_{L}+\Delta x\right)-f\left(P_{L}\right)}{f^{\prime}\left(P_{L}\right) * \Delta x}
$$

The final target function is as follows:

$$
F=\min _{R, T} \sum_{i=1}^{n} H\left(e_{i}\right)
$$

Through continuous iteration, the optimal solutions of $R$ and $T$ are finally obtained.

\section{RESULTS}

\section{A. Time synchronization results}

The communication rates of the 2D LiDAR and CCD camera are approximately $40 \mathrm{~Hz}$ and $30 \mathrm{~Hz}$, respectively. In this experiment, Rosbag was used to record the messages of Image topics and 2D point cloud simultaneously, lasting for approximately $3 \mathrm{~min}$. The ROS would automatically assign timestamps for messages received. Such timestamps are in seconds, which is consistent with the epoch time of the Unix system. The Unix time is the number of seconds that has elapsed since January 1, 1970 and does not account for leap seconds. After synchronizing the timestamp of Image Topics and 2D Point Cloud Topics with the adaptive algorithm, the communication rate was approximately $23 \mathrm{~Hz}$. Figure 7 shows the distribution of the size of sets, in which $66 \%$ is less than $0.01 \mathrm{~s}, 88 \%$ is less than $0.015 \mathrm{~s}$. And the mean value is $0.0082 \mathrm{~s}$.

\section{Timestamp Difference Distribution}

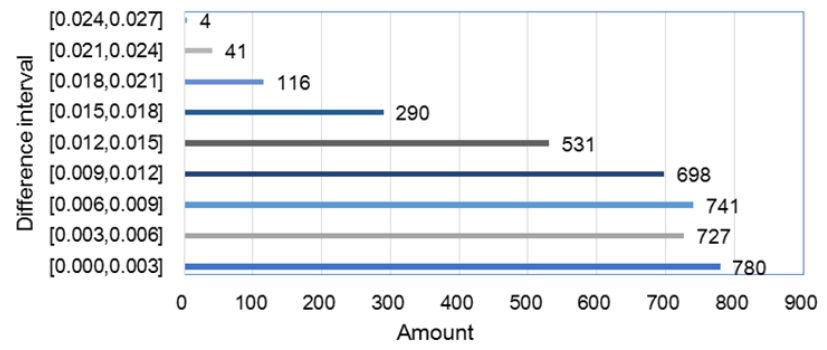

Fig.7 Difference distribution of timestamps between images and $2 \mathrm{D}$ point clouds in the same period

For further analysis, 15 continuous messages after time synchronization were randomly selected. The ROS automatic timestamp is extremely large to observe; hence, useless counting was excluded. The data are shown in Figure 8. The original timestamp of the message is extremely large to display; hence, the timestamp in Figure 8 is equal to the original timestamp minus 1587954000s. In the figure, the left coordinate axis indicates the timestamps of Image Topic and 2D Point Cloud, and the right coordinate axis shows the difference between the two timestamps. The orange and green broken lines are the timestamp changes in Image Topics and 2D Point Cloud, respectively, and the blue bar is the difference between the two timestamps. The maximum difference is $0.017 \mathrm{~s}$, as shown in Figure 8. The experimental results of time synchronization can ensure the communication frequency and satisfy the accuracy requirement.

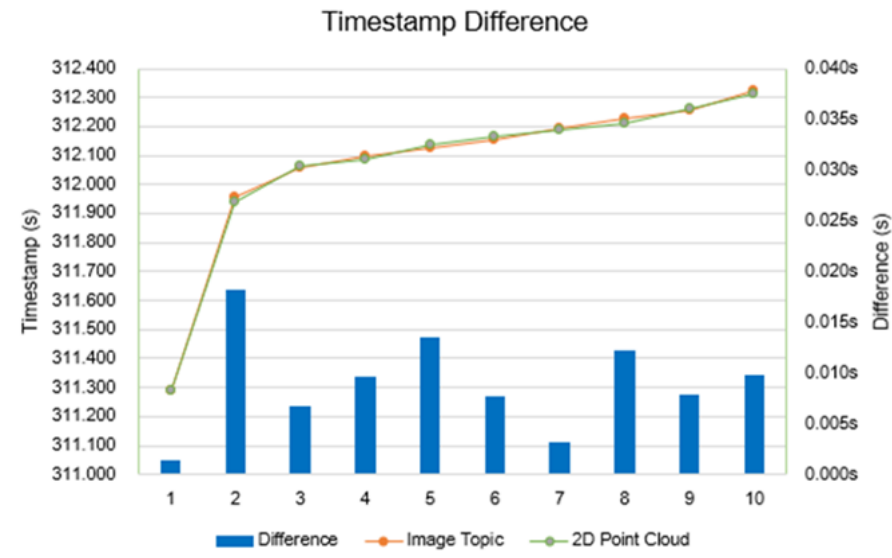

Fig.8 Timestamp difference between Image topic and 2D point cloud

\section{B. Spatial calibration results}

The external calibration experiment was completed indoors. The calibration plate was directly placed in front of the experimental platform of the forestry mobile robot. It was ensured that the scanning plane of the 2D LiDAR was in the same horizontal plane as the bulge on the calibration plate. Rosbag was used to record the images and $2 \mathrm{D}$ point cloud data simultaneously. The experimental platform was moved to ensure that the dataset contained the feature points of multiple poses. The feature points correspond to the peaks of raised cells on the calibration plate. A group of feature points of one pose is selected for illustration. When the LiDAR scans the raised cells on the calibration plate, the corner points, which are feature points, are detected in the 2D point cloud, as indicated by the red points in Figure 9. The checkboard corners are easily detected in the image. In this study, the peaks of raised cells correspond to the 2nd, 3rd, 13th, and 14th corners in the second row of the checkerboard, as indicated by the red points in Figure 10. Finally, a total of 50 datasets were selected for external calibration of the camera and 2D LiDAR. 


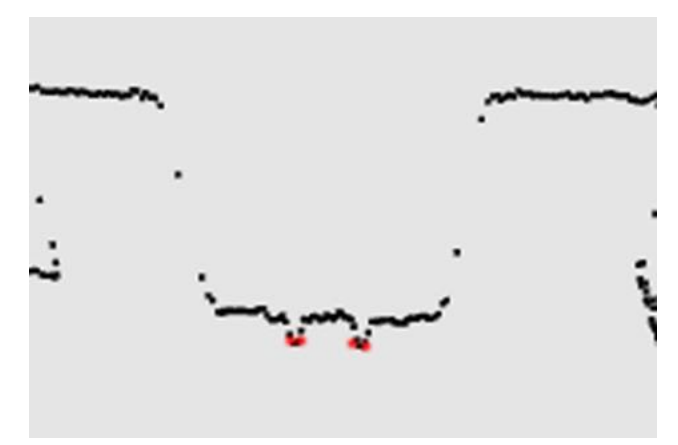

Fig.9 Corresponding feature points on the calibration board in the $2 \mathrm{D}$ point cloud

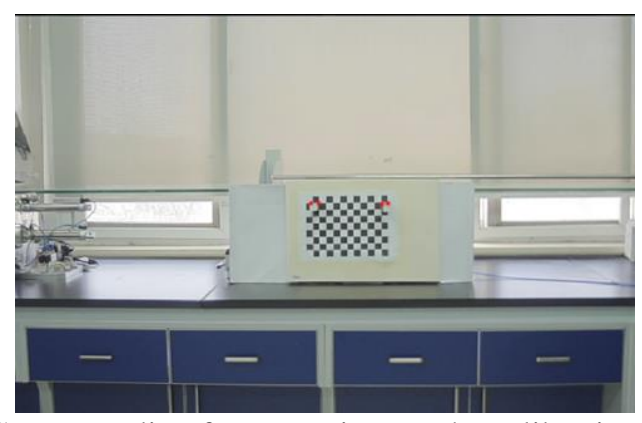

Fig.10 Corresponding feature points on the calibration board in the image

In this experiment, the camera and 2D LiDAR were vertically oriented; hence, the initial values of $R$ and $T$ are as follows:

$$
\begin{gathered}
\mathrm{R}_{\text {int }}=\left[\begin{array}{lll}
1 & 0 & 0 \\
0 & 1 & 0 \\
0 & 0 & 1
\end{array}\right] \\
\mathrm{T}_{\text {int }}=\left[\begin{array}{lll}
0 & 0 & 0.05
\end{array}\right]^{\mathrm{T}}
\end{gathered}
$$

At this point, $F_{\text {int }}=2.1829 e+06$. After 27 iterations, the final optimisation results for $\mathrm{R}$ and $\mathrm{T}$ are as follows:

$$
\begin{aligned}
\mathrm{R}_{\mathrm{fnl}} & =\left[\begin{array}{ccc}
0.0031445 & -0.99997 & -0.00714269 \\
-0.0234563 & 0.007067 & -0.9997 \\
0.99972 & 0.00331109 & -0.0234334
\end{array}\right] \\
\mathrm{T}_{\mathrm{fnl}} & =\left[\begin{array}{lll}
-0.0146695 & 0.0222217 & -0.135979
\end{array}\right]^{\mathrm{T}}
\end{aligned}
$$

At this instance, $F_{f n l}=3.029 \mathrm{e}+02$, and the gradient tolerance is the following:

$$
\frac{F_{\text {fnl }}-F_{i n t}}{F_{\text {int }}}=8.549744 \mathrm{e}-07
$$

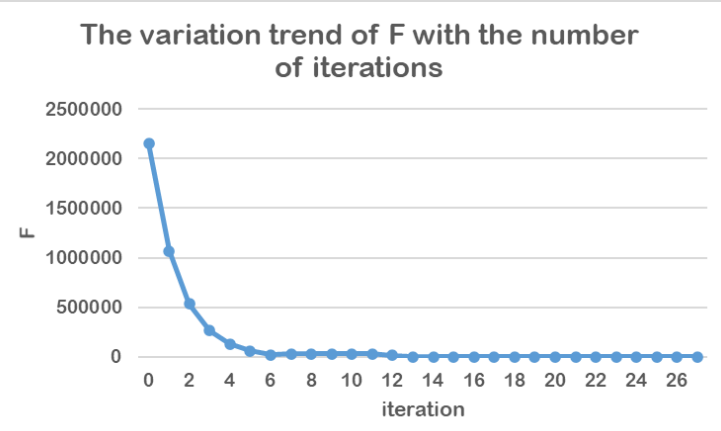

Fig.11 The change of $\mathrm{F}$ with the number of iterations

The error function (F) gradually decreases with the increase in the number of iterations, resulting in convergence, as shown in Figure 11.

Accordingly, reprojection was performed to verify the results. $2 \mathrm{D}$ point cloud was projected onto the image. Assume that the coordinate of the $2 \mathrm{D}$ point cloud is $\mathrm{C}$, and the coordinate of the corresponding image pixel is I,

$$
I=K\left(R_{f n l} * C+T_{f n l}\right)
$$

The reprojection results are shown in Figure 12. The green points are the points in the $2 \mathrm{D}$ point cloud projected onto the image. It is easy to observed that the feature points in the $2 \mathrm{D}$ point cloud and the feature points on the image overlap well. The $\mathrm{u}$-axis and v-axis average errors are is 25.30363636 and 5.19468075, respectively.

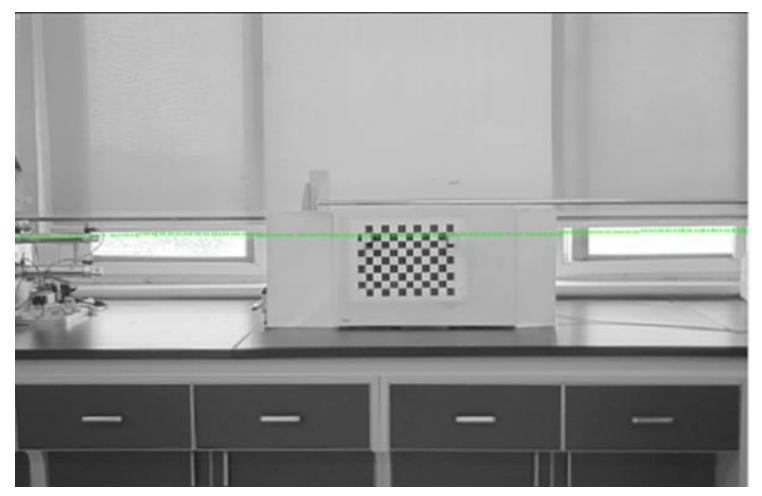

Fig.12 Reprojection result

\section{ENGINEERING APPLICATION}

The vision system of forestry mobile robot has been calibrated. The vision system can reflect 3D information around the robot in real time. Its engineering applications are discussed in this section. The data obtained by the vision system can be used for target recognition [29]-[30] as the forestry mobile robot cruises. The forestry mobile robot can recognize obstacles as it travels, and the position and size of obstacles can be determined by combining the image and 2D point cloud, ensuring its safe driving. In addition, machine learning and other methods can be applied to recognize the growth status of harvesting targets, to improve harvesting efficiency. These data can also be used for mapping and locating the forestry mobile robot. The robot builds a local map through simultaneous localization and mapping (SLAM) [31] 
and completes its own positioning using the information matching algorithm. When identifying forest roads, the 2D point cloud provides an estimate of the passable area by filtering and fitting. Moreover, machine learning and other methods are used to identify road boundaries on the image accurately. Then the passable area is projected onto the image. The relationship (expressed in terms of ratio) between the passable area and the actual road boundaries is applied to obtain the width and area of the forest road, ensuring that the work of the forestry mobile robot is accomplished. The forest road detection method is shown in Figure 13; the red line is the projection of the 2D point cloud data fitted on the image, and the white line is the road boundary on the image. Accordingly,

$$
W 2=W 1 *\left(\frac{L 2}{L 1}\right) .
$$

where: $W_{2}$ is the practical road boundary width; $W_{1}$ is the width of 2D LiDAR measurement; $L_{2}$ is the pixel value on the image corresponding to $W_{2}$; and $L_{1}$ is the pixel value on the image corresponding to $W_{1}$.

Finally, when the vision system is applied to engineering, the response speed and safety should be considered for different scenarios[32][33].

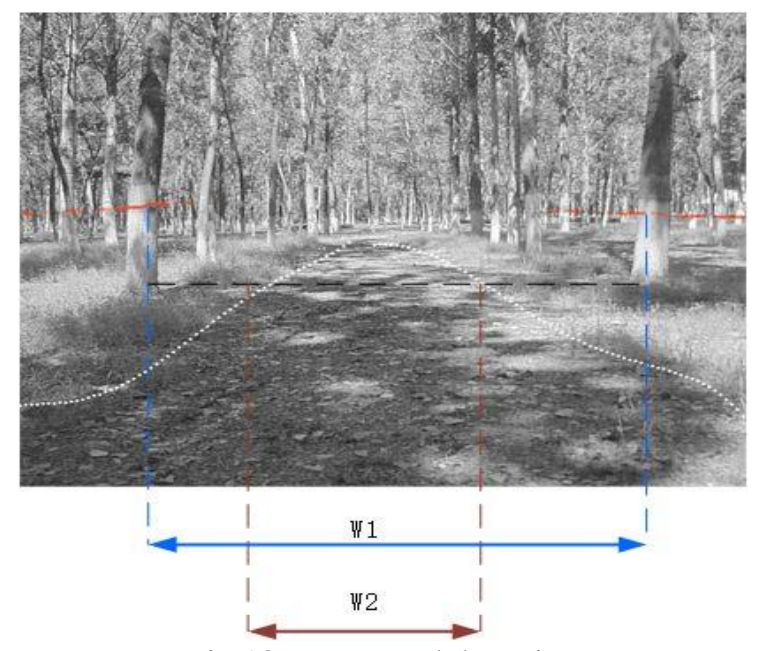

Fig.13 Forest road detection

\section{CONCLUSIONS}

To enable forestry robots to sense the rich information of the surrounding environment in real time, this paper proposes a vision system consisting of a monocular camera and 2D LiDAR. In addition, this study investigated the method for calibrating the vision system in time and space. The experimental results of time synchronization showed that the communication frequency of the combined message was $23 \mathrm{~Hz}$, and the accuracy was $0.0082 \mathrm{~s}$. The results of the space calibration experiment showed that the gradient tolerance was within 8.549744e-07, and the reprojection results were consistent with the actual states. The final joint calibration results show that this method has good robustness and accuracy. The vision system satisfies the requirements for real-time operation and accuracy of forestry mobile robots. This paper discussed the engineering application of using the system to obtain real-time 3D forest information to the forestry mobile robot. The complementarity between the $2 \mathrm{D}$ point cloud and image enabled the forestry mobile robot to achieve a more accurate recognition ability of the environment. Moreover, on the premise of ensuring accurate information, the forestry mobile robot has a lower level of data redundancy and cost than the 3D information obtained by other single sensors. Hence, this study lays the foundation for further research on forestry mobile robots and the development of forestry information intelligence systems. However, this research merely employed a camera and 2D LiDAR, which can only capture the image information of objects directly in front of the forestry mobile robot and the 2D point cloud information of a fixed height plane. Thus, it fails to fully reflect the information around the forestry mobile robot, which is anticipated to be the focus of future research.

\section{ACKNOWLEDGMENT}

This research was funded by National Key Research and Development Program of China (2019YFD1002401) and the National Natural Science Foundation of China (31670719).

\section{References}

[1] Q Yuan, S J Wu. "Forestry mechanization requires Policy technology "Two-Wheel Drive"," Woodworking Machinery, vol.03, pp.36, 2016.

[2] Y Xin. "Research on methods in dynamic obstacles detection, prediction and avoidance of autonomous vehicles," Ph.D. dissertation, University of Science and Technology of China, Hefei China, 2014.

[3] W L Huang. "Autonomous driving environment perception system construction based on ROS," Microcontrollers \& Embedded Systems, Vol.16, pp.5-9, 2016.

[4] W B Wu. "Target detection and analysis of intelligent agricultural vehicle movement obstacle based on panoramic vision," INMATEH-Agricultural Engineering, Vol. 59, pp.137-146, 2019.

[5] A S Aguiar, F N Santos, J B Cunha, H Sobreira, A J Sousa. "Localization and Mapping for Robots in Agriculture and Forestry: A Survey ", Robotics, Vol.9, 2020.

[6] Q Q Li, P Nevalainen, J P Queralta, J Heikkonen, T Westerlund. "Localization in Unstructured Environments: Towards Autonomous Robots in Forests with Delaunay Triangulation", Remote sensing, Vol.12, 2020.

[7] C L Zhang, L Y Yong, Y Chen, S L Zhang, L Z Ge, S Wang, W Li. "A Rubber-Tapping Robot Forest Navigation and Information Collection System Based on 2D Lidar and a Gyroscope", Sensors, Vol.19, 2020.

[8] C L Zhang, L Y Yong, Y Chen, S L Zhang, L Z Ge, S Wang, W Li. "A Rubber-Tapping Robot Forest Navigation and Information Collection System Based on 2D LiDAR and a Gyroscope," Sensors, vol. 19, 2019.

[9] L Y Li, J Chen J, X H Mu, W H Li, G J Yan, D H Xie, W W M Zhang. "Quantifying understory and overstory vegetation cover using UAV-based RGB imagery in forest plantation," Remote Sensing, vol. 12, 2020.

[10] A Giusti, J Guzzi, D C Ciresan, F L He, J P Rodriguez, et al. "A Machine Learning Approach to Visual Perception of Forest Trails for Mobile Robots", IEEE Robotics and Automation Letters, Vol.1, pp:661-667, 2016.

[11] J L Xue, B W Fan, J Yan, S X Dong, Q S Ding. "Trunk detection based on laser radar and vision data fusion," International Journal 
of Agricultural and Biological Engineering Vol.11, pp.20-26, 2018.

[12] M Favorskaya, A Tkacheva, I M Danilin, E M Medvedev. "Fusion of Airborne LiDAR and Digital Photography Data for Tree Crowns Segmentation and Measurement," Smart Innovation, Systems and Technologies, pp.191-201, 2015.

[13] J W Li. "Research on two-dimensional LiDAR ranging technique," Ph.D. dissertation, Huazhong University of Science \& Technology, Wuhan China, 2017.

[14] Q Zhang, R Pless. "Extrinsic calibration of a camera and laser range finder (improves camera calibration)," in Proc. 2004 IEEE/RSJ International Conference on Intelligent Robots \& Systems. Sendai, Japan, Oct 2, 2004.

[15] J Y Kim, J E Ha. " Extrinsic calibration of a camera and a 2D LiDAR using a dummy camera with IR cut filter removed", IEEE Access, Vol.8, pp:183071-183079, 2020.

[16] H V Dung, C H Danilo, K H Jo. "Simple and Efficient Method for Calibration of a Camera and 2D Laser Rangefinder," in Proc. The 6th Asian Conference on Intelligent Information and Database Systems, Bangkok, Thailand, 2014.

[17] J Briales, G J Javier. "A minimal solution for the Calibration of a 2D Laser-Rangefinder and a Camera based on Scene Corners," in Proc. IEEE/RSJ International Conference on Intelligent Robots and Systems (IROS), Hamburg, Germany, 2015.

[18] A Y Khalil, M Bassam, A W Khalid, et al. "Extrinsic Calibration of Camera and 2D Laser Sensors without Overlap," Sensors, Vol.17, pp.2346-, 2017

[19] X P Chu, J Zhou, L Chen, X B Xu. "An Improved Method for Calibration Between a 2D Lidar and a Camera Based on Point-Line Correspondences," in Proc. 2019 3rd International Conference on Artificial Intelligence, Automation and Control Technologies, Xian, China, 2019.

[20] D Kim, S Kim. "Extrinsic parameter calibration of 2D LiDAR-camera using edge matching and removal of infrared cut filter," in Proc. Signal Processing, Sensor/Information Fusion, and Target Recognition XXVIII, 2019.

[21] F Itami, T Yamazaki. "An Improved Method for the Calibration of a 2D LiDAR with Respect to a Camera by Using a Checkerboard Target," IEEE Sensors Journal, vol. 20, pp:7906-7917, 2020.

[22] Z Zhang. "A Flexible New Technique for Camera Calibration." IEEE Transactions on Pattern Analysis and Machine Intelligence, Vol.22, pp.1330-1334, 2000.

[23] J Kang. "The key technology research of multi-sensors information fusion," Ph.D. dissertation, Harbin Engineering University, Harbin China, 2013.

[24] L T Shi. "Study on time registration technology for multi-sensor information fusion," Ph.D. dissertation, National University of Defense Technology, Wuhan China, 2010.

[25] ROS Wiki. Available: http://wiki.ros.org/message_filters/ ApproximateTime.

[26] D Kalman D. "A Singularly Valuable Decomposition: The SVD of a Matrix," College Mathematics Journal, Vol.27, pp.2-23, 1996.

[27] C Jacopo, M Vittorio. "Active Regression with Adaptive Huber Loss," Computer Science. ArXiv, 2016.

[28] Wiki Pedia. Available https://en.wikipedia.org/wiki/Huber_loss.

[29] S K Zhang. "Research on obstacle detection technology based on radar and camera of driverless smart vehicles," Mh.D. dissertation, Chang'an University, Xian China, 2013.

[30] C Cappelle, E Najjar, E B Maan El, F Charpillet, D Pomorski. "Obstacle detection and localization method based on 3D model: Distance validation with ladar," in Proc. 2008 IEEE International Conference on Robotics and Automation, Pasadena CA USA, 2008.
[31] M Pierzchala, P Giguere, R Astrup. "Mapping forests using an unmanned ground vehicle with 3D LiDAR and graph-SLAM", Computers and Electronics in Agriculture, Vol.145, pp:217-225, 2018.

[32] D Rairan. "Control of a robot differential platform using time scaling", WSEAS Transactions on Systems and Control, Vol.13, pp:44-53, 2018.

[33] M O B Salem, O Mosbahi. "Towards a Safe Development of Reconfigurable Robotic Systems", WSEAS Transactions on Systems and Control, Vol.14, pp. 359-374, 2019.

Ruting Yao, born in 1995, received her Bachelor's degree from Qingdao University of Technology in 2018. Since September of 2018, she has been studying for her postgraduate degree in Beijing Forestry University. Her research interests include forestry robotics and information fusion.

Yili Zheng, born in 1981, is a professor at Beijing Forestry University. In 2009, he received his Doctor's degree from Beijing University of Posts and Telecommunications. His research interests include forestry informatization and forestry engineering equipment.

Fengjun Chen, born in1977, is an associate professor at Beijing Forestry University. In 2009, she received her Doctor's degree from University of Science and Technology Beijing. Her research interests include application of image processing and artificial intelligence in forestry.

Jian Wu, born in 1989, studied in Beijing Forestry University at 2006 2013, received his Master degree in mechanical manufacturing and automation from Beijing Forestry University in 2013. Now he works in the internship and experiment center of the school of technology in Beijing Forestry University. He is interested in researching intelligent control and intelligent detection technology.

Hui Wang is studying in Beijing Forestry University for his Bachelor's degree.

\section{Contribution of individual authors to the creation of a scientific article}

Ruting Yao executed the experiments and carried out the engineering application.

Yili Zheng analyzed the human resource allocation problem and carried out the simulation and the optimization.

Fengjun Chen provided technical guidance.

Jian Wu optimizated the algorithm.

Hui Wang implemented the Algorithm.

\section{Creative Commons Attribution License 4.0 (Attribution 4.0 International, CC BY 4.0)}

This article is published under the terms of the Creative Commons Attribution License 4.0

https://creativecommons.org/licenses/by/4.0/deed.en_US 